\title{
Metaphern als stilistische Textelemente in den Leitartikeln des Mährischen Tagblatts
}

\author{
Metaphors as Stylistic Text Elements in the Editorials of the Daily \\ Mährisches Tagblatt
}

Michaela Kaňovská

\begin{abstract}
The paper deals with the use of metaphors in newspaper editorials. Using the results of Christa Baldauf's study (1997) as a basis, it gives a short overview of the kinds of conceptual metaphors in a sample of 20 editorials in the daily Mährisches Tagblatt published in the last quarter of the $19^{\text {th }}$ century. Afterwards, it focuses on the description of the conceptual metaphor POLITICS IS WAR / BATTLE. Attention is paid to the expressions themselves and their position in the text.
\end{abstract}

\section{Keywords}

(conceptual) metaphor, political communication, editorial, German in Moravia, Mährisches Tagblatt 
Der vorliegende Beitrag befasst sich mit der Metaphorik in einer der meinungsbetonten Pressetextsorten, dem Leitartikel. Die Pressetextsorten werden in der Regel nach ihren intentionalen Funktionen (Informieren, Überzeugen oder Unterhalten) eingeteilt (vgl. Malá 2009: 127; Rykalová 2008: 13-19). Die meinungsbetonten Textsorten unterscheiden sich von den informationsbetonten vor allem dadurch, dass in ihnen (direkt oder indirekt) die persönliche Meinung, Einstellung des Journalisten zum Ausdruck kommt. Dazu werden häufig verschiedene emotional-expressive Sprachmittel verwendet, zu denen auch Metaphern gehören - die kreativen, innovativen sowie die schon etablierten, konventionalisierten, von denen vor allem die lexikalisierten Mehrwortmetaphern, d. h. metaphorische Phraseme, stilistisch wirksam sind (vgl. Burger 2005: 215; Fleischer 1997: 30-33, 218-221, 224; Gehr 2014: 24-27; Küster 1983: 37; Lüger 1995: 67-70, 133136, 138, 141; Malá 2009: 128; Rykalová 2008: 49).

Im Folgenden werden nach einer Charakteristik des untersuchten Materials, einer kurzen Darstellung der traditionellen und der kognitiven Metaphernauffassung und der Klassifikation von Metaphern die Ergebnisse ausgewählter Untersuchungen zu Typen und Funktionen von Metaphern in journalistischen Texten präsentiert und anhand Beispiele (d. h. eher implizit) mit den in den Leitartikeln des Mährischen Tagblatts (=MT) verwendeten Metaphern verglichen. Dann wird von den in diesen Texten versprachlichten Metaphernmodellen die aufgrund der Aufgabe des $M T$ (mehr s. u.) am ehesten zu erwartende konzeptuelle Metapher POLITIK IST KRIEG / KAMPF mit ihren stilistisch wirksamen Sprachmetaphern näher vorgestellt. Dabei wird auch der Position der Metaphern in der Textstruktur Aufmerksamkeit geschenkt, weil damit bestimmte Funktionen zusammenhängen wie Kontaktherstellung (in einer Überschrift), Textrahmung und Textgliederung (am Anfang und am Ende eines Textes bzw. eines Abschnittes), Bewertung (z. B. im zentralen Teil oder am Schluss), im Vordergrund steht hier allerdings die „Inventarisierung“ der sprachlichen Metaphern in diesen Texten.

\section{Charakteristik der Leitartikel im Mährischen Tagblatt}

Als Material für die Untersuchung dienten 20 Leitartikel des $M T$ aus der Jahren 1880 bis 1893, die die politische, ökonomische, soziale u. a. Situation in Österreich-Ungarn zur Zeit der konservativen Regierung des Grafen Eduard von Taaffe (1879-1893) kommentieren. ${ }^{1}$ Der Antritt dieser Regierung gab nämlich Impuls zur Gründung dieses deutschliberalen Blattes, das von 1880 bis 1945 sechsmal wöchentlich erschien, bis zum 30.11.1938 in Olmütz, danach in Brünn. Es war Nachfolger des Deutschen Volksblatts für Mähren, das im Jahre 1872 vom Rechtsanwalt Dr. Jakob Eben als eine unabhängige (Wochen-) Zeitung der im Deutschen Verein für Mähren versammelten Liberalen gegründet wurde. Dr. Eben war Berater des Olmützer Bürgermeisters Josef Engel für politische

1 Das Korpus wurde primär für die Untersuchung der Phrasemverwendung zusammengestellt (zu Kriterien der Textauswahl vgl. Kaňovská 2015). Die Untersuchung der Metaphern stellt Weiterführung eines der Teilaspekte dar. Im Prinzip ist jedes Jahr mit einem oder zwei Leitartikeln vertreten. Die übrigen MT-Leitartikel eines „übergeordneten“ Teilkorpus von 163 Texten derselben Zeitspanne wurden, wenn nötig, mitberücksichtigt. 
Angelegenheiten und seine Zeitung wurde zum offiziellen Blatt des Olmützer Rathauses (vgl. Klenovský - Papoušek 1997-1998: 28-30; Föhner 1930: 137-139; Nešpor 1998: 287). Zur Erfüllung der Aufgabe des $M T$ - die deutsche Bevölkerung, Kultur und Sprache in der Monarchie zu verteidigen und zu fördern - wurden besonders die Leitartikel benutzt, was mit der meinungsbildenden Funktion dieser Textsorte zusammenhängt (vgl. Lüger 1995: 69, 126-136; Brinker 1997: 106, 109).

Der Leitartikel wird als die „Flagge der Zeitung“ bezeichnet, da er die Meinung der Redaktion, die Tendenz der Zeitung wiedergibt. In einer Ausgabe hat er einen festen Platz, meistens auf der Titelseite (so auch im MT), oft aber auch im Innern der Zeitung. Normalerweise hat der Leitartikel täglich die gleiche Länge, die untersuchten MT-Texte hatten allerdings wechselnden Umfang von 3 bis 5 Spalten unterschiedlicher Länge (92 bis 207 Zeilen bzw. 597 bis 1433 Wörter). Der Leitartikel kommentiert die Entwicklungen in der Gesellschaft, er nimmt viel eindeutiger Stellung zu einem bedeutenden Thema als Kommentare und bezieht verschiedene Zusammenhänge in seine Argumentation ein (vgl. Mast (Hrsg.) 1994: 190; Reumann 2004: 145-146; Stöber 2005: 190). Es ist allerdings zu berücksichtigen, dass es mehrere Typen von Leitartikeln gibt. Der Zeitungswissenschaftler Dovifat, dessen Gliederung bei der Untersuchung der MT-Texte angewandt wurde, unterscheidet z. B. (1) den kämpfenden, (2) den stellungnehmenden und begründenden, (3) den erläuternden und unterrichtenden, (4) den rückschauenden, (5) den Gedächtnis-, (6) den vorschauenden und (7) den betrachtenden Leitartikel (vgl. Dovifat 1967: 141). Diese verschiedenen Formen kommen im MT meist gemischt vor. ${ }^{2}$

Da der Leitartikel eine Sonderform des Kommentars ist, sind auch für seine Struktur fünf Elemente typisch: (I) Die Überschrift (= Ü), die das Anliegen des Artikels thematisieren kann, aber (als Leseanreiz) nicht eindeutig sein muss und meist wertende Elemente enthält. (II) Die Texteinleitung (= E), die zusammen mit dem Schluss oft einen Rahmen bildet, der die zentrale Wertung betont. Hier informiert der Autor kurz über den zugrunde liegenden Sachverhalt, die Darstellung ist aber schon selektiv, sie stützt indirekt die dominierende Sprachhandlung (Bewertung, ggf. explizite Aufforderung) in dem zentralen Textteil. Außerdem soll die Texteinleitung die Aufmerksamkeit des Lesers fesseln und ihn zum Weiterlesen anregen. (III) Der Hauptteil, d. h. der argumentative Kern (= A), in dessen Zentrum i. d. R. eine bestimmte Bewertung $(=\mathrm{ZW})$ steht. Unterstützende Handlungen (Rechtfertigungen, Begründungen, Erläuterungen) sollen den Leser von der Richtigkeit bestimmter Aussagen des Autors überzeugen und die Bewertungsübernahme und dementsprechendes Verhalten unterstützen. Die Aufforderung zu einer der präsentierten Meinung entsprechenden Handlung kann auch explizit ausgedrückt werden (im Imperativ, mit den Modalverben sollen, müssen, vgl. Lüger 1995: 70-71). (IV) Die fakultative Präsentation einer Gegenposition $(=G)$ und ihre argumentative Widerlegung

2 Für Leitartikel als Sonderform von Kommentaren könnten auch die für Kommentare erstellten Typologien verwendet werden. La Roche (1992: 154) unterscheidet z. B. den Geradeaus-, den Argumentations- und den Einerseits-andererseits-Kommentar. Nowag und Schalkowski sehen den erklärenden und den (be)wertenden Kommentar als zwei Idealtypen, die in der Praxis in verschiedenen Mischformen zu finden sind, und nach der Strategie des Kommentators beim Umgang mit argumentativen und erklärenden Inhalten unterscheiden sie den Standpunktkommentar, den diskursiven bzw. diskursiv ausschließenden Kommentar und den dialektischen Kommentar (vgl. Nowag - Schalkowski 1998: 46-52, 163-176). 
(= GW), die die Gültigkeit der dominierenden Bewertungshandlung stärken soll. (V) Der Schluss (=S), wo der Autor die wichtigsten Gedanken zusammenfasst und Folgerungen zieht, den Text mit einer zu weiterem Nachdenken anregenden Bemerkung, mit einer ironischen Pointe, ggf. mit einem Appell endet (vgl. Lüger 1995: 126-136).

\section{Metaphern}

Metapher wird definiert als Bedeutungsübertragung von einem Gegenstand auf einen anderen aufgrund der äußeren Ähnlichkeit (vgl. Malá 2009: 57). Bei Metaphorisierung wird die Bedeutung eines Wortes oder einer Wortgruppe aus einem Herkunftsbereich auf einen Zielbereich übertragen (vgl. Gehr 2014: 18; Kohl 2007: 9, 19). Aufgrund gemeinsamen Wissens unterscheiden die Sprecher einer Sprachgemeinschaft (a) kreative, innovative, okkasionelle, poetische Metaphern und (b) konventionelle, schon etablierte, usuelle Metaphern, bei denen man noch die „lebendigen“ Metaphern von den „toten“, verblassten Metaphern trennen kann; die letzteren wie (Stuhl-)Bein werden nicht mehr als Metaphern wahrgenommen, da sich die Sprecher nicht mehr der Dualität der nicht-wörtlichen und wörtlichen Verwendung bewusst sind (vgl. Baldauf 1997: 15; Keller-Bauer 1983: 57-59; Kohl 2007: 20). Die konventionellen Metaphern (z. B. negative Tiermetaphorik wie Ratten, Ungeziefer) können nicht nur über den wörtlichen Gebrauch verstanden werden, sondern auch über frühere metaphorische Verwendungen (in diesem Fall nicht nur im NS-Regime, sondern schon bei Luther, später bei Schiller u. a., vgl. Keller-Bauer 1983: 47, 49-55, 57). Den sog. toten Metaphern wird als Metaphern der Alltagssprache (Alltagsmetaphern) Aufmerksamkeit in der kognitiven Metapherntheorie geschenkt (vgl. Baldauf 1997: 15; Keller-Bauer 1983: 58; Lakoff - Johnson 2002: 15).

\subsection{Metaphern in der kognitiven Linguistik}

In der kognitivlinguistischen Auffassung wird die Metapher als Prinzip menschlicher Wahrnehmung und Wissensorganisation verstanden. Im Falle der sog. konzeptuellen Metaphern besteht die Metaphorisierung in der Projektion eines Ausgangsbereichs auf einen Zielbereich. Dieser Vorgang stellt einen Typ der kognitiven Modelle dar (vgl. Lakoff 2006: 78). Ein abstraktes kognitives Metaphernmodell kann durch mehrere konzeptuelle Metaphern spezifiziert werden, bei denen wieder Ebenen verschiedenen Abstraktionsgrades zu unterscheiden sind. Zum Beispiel beim metaphorischen Phrasem jmdm. Sand in die Augen streuen, jmdm. etw. vormachen, jmdn. vorsätzlich täuschen oder betrügen' kann die Verbindung zwischen den konzeptuellen Strukturen angesiedelt werden auf einer abstrakteren Ebene (TÄUSCHUNG IST STÖRUNG DES SEHVERMÖGENS) oder auf einer konkreteren Ebene. Das Ausgangskonzept STÖRUNG DES SEHVERMÖGENS wird in diesem Fall durch eine gewaltsame Handlung, Verletzung der Augen angedeutet, im Unterschied zum Phrasem jmdn. hinters Licht führen, wo es durch Entzug von Licht, mit dem Resultat der Dunkelheit, Unfähigkeit der Orientierung 
geschieht. Beide Phraseme und weitere metaphorische Ausdrücke lassen sich dann aufgrund abstrakter konzeptueller Entsprechungen zwischen Gruppen von Ausgangs- und Zielbereichen zu dem Metaphernmodell WISSEN IST SEHEN bzw. WISSEN IST LICHT (antonymisch NICHT WISSEN IST NICHT SEHEN / NICHT WISSEN IST DUNKELHEIT) zusammenfassen (vgl. Dobrovol'skij - Piirainen 2009: 20-21). Sprachlich wird eine konzeptuelle Metapher in einer Einwortmetapher (einem einfachen Wort oder einer Wortbildungskonstruktion) oder einer metaphorischen Verbindung realisiert. So liegt z. B. das obige Metaphernmodell den Ausdrücken (lexikalischen Metaphern) etw. (aus etw.) ersehen, politischer Weitblick (vs. politische Kurzsichtigkeit), jmdm. gehen die Augen auf u. a. zugrunde (vgl. den MT-Leitartikel vom 12.6.1883; Malá 2009: 65-66; Burger 2010: 90). Ein Konzept kann mit mehreren konzeptuellen Metaphern verbunden sein (z. B. LEBEN ist WERTVOLLER BESITZ, BEHÄLTER, REISE, SPEISE, SPIEL, WEG u. a.) und eine lexikalische Metapher kann durch mehrere konzeptuelle Metaphern motiviert sein (z. B. das metaphorische Phrasem mit dem / seinem Leben spielen ist durch die konzeptuellen Metaphern LEBEN IST GLÜCKSSPIEL und LEBEN IST WERTVOLLER BESITZ motiviert, vgl. Kispál 2013: 63, 111, 262-265).

Im Unterschied zu den Untersuchungen, bei denen zu einem abstrakten Zielbereich die Ausgangsbereiche und die entsprechenden metaphorischen Ausdrücke gesucht werden, wurden kognitivlinguistische Untersuchungen von metaphorischen Ausgangsbereichen (mindestens in Bezug auf metaphorische Phraseme) bisher nur vereinzelt durchgeführt (z. B. die Komponenten Feuer und Hand in Kövecses - Szabó 1996: 329-345, Hand in Hegedüs-Lambert 2010; vgl. Kispál 2013: 59, 75).

\subsection{Klassifikation der konzeptuellen Metaphern}

Die Begründer der kognitiven Metapherntheorie Lakoff und Johnson teilen die konzeptuellen Metaphern in drei Gruppen ein und unterscheiden: (a) die Orientierungsmetaphern, die auf menschlicher Raumerfahrung beruhen (z. B. GUT IST OBEN: mit erhobenem Haupt), (b) die ontologischen Metaphern, die Abstraktes mithilfe vom Konkreten (Entitäten oder Substanzen) zu verstehen erlauben (z. B. ABSTRAKTA SIND DINGE: das Leben in die Hand nehmen; ÄRGER IST HITZE: vor Wut kochen), und (c) die strukturellen Metaphern, die ermöglichen, verschiedene Aspekte desselben Szenarios darzustellen (z. B. LEBEN IST EINE REISE: im gleichen Boot sitzen, vgl. Baldauf 1997: 20-28; Malá 2009: 70; Lakoff - Johnson 2002: 26-34, 39-48, 79-85; Schmitt 2004: 6-8). Dem letztgenannten Szenario entsprechend ist dann die Person, die das Leben führt, der Reisende, Schwierigkeiten im Leben sind die Hindernisse bei der Reise, Entscheidungen im Leben sind Kreuzwege usw. (vgl. Kispál 2013: 62). Andererseits liegen solchen Metaphern bestimmte Bildschemata zugrunde, die ihre innere Strukturierung und gemeinsame Aspekte verschiedener konzeptueller Metaphern herausstellen, z. B. die konzeptuelle Metapher LEBEN IST EINE REISE kann auf das WEG-Schema zurückgeführt werden, oder das ZYKLUS-Schema prägt die konzeptuellen Metaphern LEBEN IST EIN TAG oder EIN JAHR (z. B. der Lebensabend; der Frühling des Lebens; vgl. Baldauf 1997: 
66-71; Kispál 2013: 61, 263; Lakoff 2006: 265-270; Malá 2008: 48). Für Metaphern, die nur mithilfe außersprachlichen, oft kulturgebundenen Wissens adäquat interpretierbar sind, verwenden Dobrovol'skij und Piirainen (2009: 24-25) den Terminus framebasierte Metaphern. Die Notwendigkeit der Kenntnis des entsprechenden Frames für die Interpretation wird besonders deutlich bei den heute für die meisten Sprecher „undurchsichtigen“ Metaphorisierungen, z. B. beim Phrasem jmdm. den Korb geben die Kenntnis des Frames FREIERN (wie es in früheren Zeiten üblich war, vgl. D11: 441; Burger 2010: 69-70; Palm 1995: 13).

Die Klassifikation der Alltagsmetapher von Lakoff und Johnson wurde von Baldauf (1997) durch eine Klassifikation ersetzt, die als einheitliches Kriterium die Art und Struktur des jeweiligen Herkunftsbereichs verwendet. Nach zunehmender struktureller Komplexität unterscheidet Baldauf (1) Attributsmetaphern - Projektion von physisch wahrzunehmenden, wertenden Eigenschaften auf Personen, Objekte oder Sachverhalte, auf deren abstrakte Eigenschaften so Bezug genommen wird (z. B. MANGEL AN EMOTIONEN IST KÄLTE: gefühlskalt). Mit (2) ontologischen Metaphern werden abstrakte Bereiche als Objekt oder Substanz konzeptualisiert und sie werden dann durch die anderen Metaphernklassen spezifiziert (z. B. ABSTRAKTA SIND DINGE: Maßnahmen ergreifen). Die weiteren Klassen sind (3) bildschematische Metaphern, d. h. Projektion gestalthafter, bildschematischer Struktur in abstrakte Bereiche (z. B. EMOTIONEN SIND BEḦ̈LTER: in Aufregung sein) und (4) Konstellationsmetaphern - Projektion ganzer, gestalthafter Konstellationen in abstrakte Bereiche (z. B. POLITIK IST KRIEG: Wahlkampf, vgl. Baldauf 1997: 83-84, 91-92). Wenn POLITIK als KRIEG konzeptualisiert wird, sind nach diesem Szenario Parteien Armeen, die an verschiedenen Fronten kämpfen, sie werden als militärische Einheiten strukturiert: Politiker, die nach ihrer Funktion oder Einsatzgebiet als Streiter, Wahlkämpfer, Frontmann, Alarmist u. a. bezeichnet werden, bilden zusammen eine Truppe, die in kleinere Einheiten wie Kompanien oder Verbände organisiert ist. Es gibt aber auch über die Parteien hinausgehende Organisationsstrukturen, die Mitglieder einer Partei befinden sich z. B. in einem Lager usw. (vgl. Kudla 2008: 76-78, 105).

\subsection{Sonderfälle von Metaphern und andere als metaphorische Motivationstypen}

Dobrovol'skij - Piirainen (2009) erwähnen bei der Behandlung von metaphorischen Ausdrücken unter kognitivem Aspekt noch zwei Sonderfälle: Erstens Kinegramme, d. h. konventionalisierte Verbalisierungen von nonverbalem Verhalten, die einerseits eine kinetische, andererseits eine symbolische, kulturell kodifizierte Bedeutung haben (z. B. die Achseln zucken ,mit einem Hochziehen der Schulter zu verstehen geben, dass man etw. nicht weiß od. nicht versteht', vgl. DU; Dobrovol'skij - Piirainen 2009: 28). Zweitens geht es um das usualisierte Wortspiel, das z. B. auf phonischen Elementen beruhen kann: die Verbindung zwischen den konzeptuellen Ebenen des Idioms etw. aus der Armenkasse kriegen (bes. berlin., scherzh., verhüll.) ,Prügel bekommen“ kommt aufgrund von lautlichen Assoziationen zustande (Arme, d. h. arme Menschen, und die Arme, mit denen Prügel 
ausgeteilt werden, vgl. D11: 60; Dobrovol'skij - Piirainen 2009: 29, 102-114; Fleischer 1997: 99).

Für die Beschreibung der Metaphern im untersuchten Text-Korpus ist weiter noch die Abgrenzung der metaphorisch motivierten Ausdrücke von den metonymisch, symbolisch, intertextuell und indexal motivierten wichtig. Die Metonymie ist traditionell eine Bedeutungs- bzw. Bezeichnungsverschiebung aufgrund kausaler, räumlicher, zeitlicher, instrumentaler u. a. Zusammenhänge, z. B. Weißes Haus (Ort) steht für ,die US-Regierung، (eine Institution, vgl. Malá 2009: 58, 72). In kognitivlinguistischen Untersuchungen wurde gezeigt, wie bei metonymischen Phrasemen zwei Szenarios in Beziehung zueinander gesetzt werden, um Anschaulichkeit, Expressivität zu erhöhen (vgl. Hartmann 1999: 227-228, 233-234). Beim Phrasem seinen Hut nehmen müssen ,zurücktreten müssen, aus dem Amt scheiden müssen“ tritt z. B. „das Element den Hut nehmen aus dem Szenario ,Aufenthalt in einer menschlichen Behausung، (aus dem Slot, Verlassen der menschlichen Behausung, Vorbereitungen dazu') durch [...] Übertragung an die Stelle des Elements ,Aufgeben der beruflichen Position“ aus dem Szenario ,eine berufliche Position, ein Amt innehaben““ (Hartmann 1999: 232). Metaphorische und metonymische Prozesse werden also auch kombiniert (vgl. Burger 2010: 85-86; Malá 2009: 72-73).

Die symbolische Motivation ist die Motivation aufgrund des semiotischen Wissens über die Bedeutung eines Symbols in kulturellen Kenntnissystemen außerhalb der Sprache. Symbolisch motiviert ist z. B. das Simplex Fuchs ,schlaue, listige Person' oder das Phrasem Gold in der Kehle haben ,eine besonders schöne Singstimme haben' (vgl. Dobrovol'skij - Piirainen 2009: 30-31; Piirainen 2007: 212-214). Im Falle der intertextuellen Motivation erschließt sich die Bedeutung der Lexikoneinheit v. a. aufgrund eines bestimmten Textwissens. Die Ausdrücke gehen auf bereits bestehende Texte zurück, z. B. das Phrasem sich mit fremden Federn schmücken, Verdienste anderer als die eigenen ausgeben (und sich damit brüsten)' stammt aus einer Fabel von Äsop (und wurde in zahlreiche Nachdichtungen übernommen, vgl. DU; Piirainen 2011: 172; Röhrich 2004: 423). Da die Bekanntheit der zugrunde liegenden Texte graduell ist, können manche intertextuell motivierte Ausdrücke von den weniger literarisch bewanderten Sprachteilhabern metaphorisch oder symbolisch gedeutet werden (vgl. Dobrovol'skij - Piirainen 2009: 32-36). Die indexale Motivation basiert auf phonischen bzw. konzeptuellen Analogien. Im Falle der phonischen Verweise weisen bestimmte Merkmale der lautlichen Struktur eines Ausdrucks auf entsprechende Merkmale seiner Bedeutung hin, z. B. (österr.) nach Melk gehen ,sich übergeben‘. Bei pragmatischen Verweisen, z. B. dem Phrasem dann bin ich der Kaiser von China ,das glaube ich nicht, was du da sagst', ist die Absurdität der Aussage mit der Phrasembedeutung indexal verbunden (vgl. Dobrovol'skij - Piirainen 2009: 36-38).

In ein und demselben Ausdruck können allerdings verschiedene Motivationstypen auftreten, z. B. beim Phrasem im siebten Himmel sein (1) die metaphorische Motivation - einerseits die konzeptuelle Metapher GLÜCKSEMPFINDUNG IST RÄUMLICHE HÖHE bzw. GUT IST OBEN, andererseits die framebasierte Metapher, deren Grundlage das Weltwissen über den Frame Himmel (der Himmel als Ort des Glücklichseins) ist; (2) symbolische Motivation - der Symbolwert von SIEBEN als ,gut, viel bzw. sehr', d. h. der siebte Himmel ,der besonders gute Himmel'; ggf. - für Spezialisten - auch (3) intertextuelle 
Motivation, vgl. die Lehre von den sieben Himmeln im Talmud, Koran usw. (vgl. Dobrovol'skij - Piirainen 2009: 19, 39-40).

\section{Metaphern in journalistischen Texten}

\subsection{Festgestellte Metapherntypen}

Die oben erwähnten und andere Klassifikationen wurden für die Beschreibung des Metapherngebrauchs in verschiedenen Texttypen, darunter auch in journalistischen Texten, verwendet. Reger (1980) unterscheidet z. B. aufgrund von stilistischen Funktionen (z. T. mit Berücksichtigung metaphorischer Herkunftsbereiche) (a) dynamisierende Metaphern (z. B. die Fußballspieler jagen oder feuern den Ball), (b) konkretisierende Metaphern (Naturmetaphern wie die Berg-Metapher Gipfeltreffen; Weg-, Haus- oder Schiffs-Metaphorik u. a.); (c) personifizierende Metaphern (z. B. die Welt atmet auf; der Kaiser der Kicker als Periphrase für Beckenbauer) und (d) sensorische Metaphern (z. B. in glänzender Form, hart kritisieren; vgl. in Bezug auf Qualitätszeitungen Reger 1980: 79-94; Malá 2009: 59, 62-63). Gehr, der die Metaphern und Phraseme in politischen Kommentaren untersucht hat, erwähnt zuerst ihre grammatische Klassifikation (Substantiv-, Adjektiv- und Verbmetaphern, vgl. Gehr 2014: 19; Kohl 2007: 46-50). Im Zusammenhang damit wären die morpho-syntaktischen Klassen von metaphorischen Phrasemen zu erwähnen (vgl. Burger 2010: 42-45; Fleischer 1997: 138-161), z. B. Spitze des Eisbergs; kurz angebunden; mit Pauken und Trompeten; die Segel streichen (vgl. Gehr 2014: 22-27).

Zweitens verwendet Gehr die schon bei Reger erwähnte Gruppierung der Metaphern nach ihrem Herkunftsbereich. Zu den häufigsten Herkunftsbereichen ${ }^{3}$ der Metaphern in journalistischen Texten, v. a. in politischen Kommentaren ${ }^{4}$, gehören nach ihm (a) Sport (z. B. Fußball: jmdn. ins Abseits stellen, vgl. auch Küster 1983: 44-45)5 ; (b) Militärwesen (Wahlkampf; jmdn. aufs Schild heben, vgl. Baldauf 2007: 213-231; Kudla 2008); (c) der menschliche Körper (auf die Nase fallen); ${ }^{6}$ (d) Verkehrswesen (Straßenverkehr: Ampelkoalition; Schiffsverkehr: am Ruder stehen, vgl. Baldauf 2007: 210-212; Küster 1983: 42; Bahnverkehr: Entgleisung, vgl. Baldauf 2007: 210; Flugverkehr: im Sinkflug sein) und

3 Gehr verwendet die Bezeichnung „thematische“ bzw. „metaphorische Wortfelder“ (vgl. Gehr 2014: 33). In den politischen Kommentaren zum Landtagswahlkampf in Nordrhein-Westfalen 2010 stellte er in zwei Boulevard- und drei Qualitätszeitungen insgesamt 46 Herkunftsbereiche fest (vgl. Gehr 2014: 79ff., 93, 101ff., 116ff.).

4 Zu Herkunftsbereichen in journalistischen Wirtschaftstexten vgl. Lüger (1995: 38); Duhme (1991: 139155); Höppnerová (2006: 234-236) u. a.

5 Gehrs Bestimmung des Herkunftsbereichs geht eher auf Assoziationen der heutigen Sprecher zurück. Phraseme wie jmdm. den Wind aus den Segeln nehmen, die Segel streichen (bei Gehr „Segelsport“) werden in den Wörterbüchern auf die Seefahrt zurückgeführt (vgl. D11: 698, 873) oder die von Gehr dem „Pferdesport“ zugeordneten Phraseme wie die Zügel aus der Hand geben nehmen Bezug auf Pferde als Nutztiere, Fortbewegungsmittel in früheren Zeiten (vgl. Röhrich 2004: 1566, 1778; D11: 740).

6 Menschliche Körperteile kommen oft in Metaphern für psychische oder physische Zustände vor (vgl. Malá 2009: 71; Burger 2010: 92-93 u. a.). 
damit zusammenhängend (e) der Weg (den richtigen Weg einschlagen, vgl. Baldauf 1997: 67-68, 139-150; Molly 2002: 356-357, 365-393); (f) Meteorologie (Sturm der Entrüstung auslösen); (g) Kultur (Theater und Film: unter jmds. Regie; hinter den Kulissen, vgl. Baldauf 2007: 187-188; Kudla 2008: 88-90; Küster 1983: 37-39; Musik: die erste Geige spielen); (h) Handwerk (den Nagel auf den Kopf treffen); (i) Religion (Prophet); (j) Zoologie (Federn lassen); (k) Medizin - u. a. Krankheit (das politische Infarktrisiko, vgl. Baldauf 1997: 206-208), weiter auch die Herkunftsbereiche (1) (allgemeine) Bewegung (über etw. achselzuckend hinweggehen; wackeln, vgl. Küster 1983: 42-44) oder (m) Spiel (v. a. Karten- oder Schachspiel: die besten Karten haben; eine lange Hängepartie, vgl. Baldauf 2007: 184-186; zu allen Bereichen vgl. Gehr 2014: 34-63, 116-132; Küster 1983: 39-41; Polenz 1991: 55; Reger 1980: 80-89). Polenz erwähnt außerdem noch (n) die Jagdmetaphorik (jmdm. auf den Leim gehen, vgl. Polenz 1991: 56). Skirl und Schwarz-Friesel führen als Beispiel für eine innovative Konzeptualisierung der Politik (o) die Nahrung an (vgl. Skirl - Schwarz-Friesel 2007: 77, zit. nach Mutz 2010: 111). ${ }^{7}$ Bei der Formulierung eines Gedankens kann der Journalist Metaphern aus einem oder aus mehreren Herkunftsbereichen (homogene oder heterogene Sprachbilder) verwenden. ${ }^{8}$ Dabei hat er im ersten Fall auf die Gefahr der Übertreibung, der moralischen Inadäquatheit, der Meinungsverschleierung oder Belanglosigkeit, im zweiten Fall v. a. auf die Gefahr des Bildbruchs zu achten (vgl. Gehr 2014: 64-72; Küster 1978: 106; Küster 1983: 44-45). Ein Autor kann in seinen politischen Texten auch Metaphern bestimmter Herkunftsbereiche bevorzugen (z. B. Václav Klaus Familien-Metaphern für die Nation oder Kampf- und Religionsmetaphern für den Klimawandel, vgl. Fedina 2011: 6-12).

Nach Baldauf oder Niehr sind es die Kampfmetaphern, die im politischen Diskurs von herausragender Bedeutung sind (vgl. Baldauf 1997: 214-215; Niehr 2014: 146; weiter Kudla 2008: 2-3, 113-115; Küster 1978: 74-75; Polenz 1991: 56; Reger 1980: 85). Einige dieser Metaphern können noch in einem anderen Bildspenderbereich angesiedelt werden, z. B. Verteidiger im Sport, aber auch im juristischen Kontext - in einer Gerichtsverhandlung, wodurch bei Textrezeption der Eindruck entstehen kann, dass in dem Kampf bestimmte prozedurale Spielregeln beachtet werden müssen (vgl. Küster 1978: 82-83). Küster, der die militärische Metaphorik in den Leitartikeln der Welt und der Süddeutschen Zeitung (=SZ) untersuchte, stellte fest, dass diese Metaphern am meisten in den innenpolitischen Leitartikeln verwendet werden - in der Welt zweimal, in der SZ fast dreimal häufiger als in den außenpolitischen Leitartikeln (vgl. Küster 1978: 89-90). Sonst hängt die Metaphorik des politischen Diskurses von dem jeweiligen Thema ab: z. B. im Migrationsdiskurs der 1960er Jahre war es die Wasser-Metaphorik (vgl. Molly 2002: 359-360; Niehr 2014: 146-147, 164).

Bei der Untersuchung von Metaphern in konkreten Texten werden neben den Herkunftsbereichen auch die versprachlichten kognitiven Metaphernmodelle gelegentlich

7 Solche Metaphern kommen aber auch schon im MT vor, vgl. den Leitartikel „Gesegneter Appetit“ (18.8.1880).

8 Burger hat festgestellt, dass nicht-phraseologische Metaphern in einem stark metaphorischen Text eher homogen und kohärenzstiftend sind, während metaphorische Idiome verschiedene Konzepte versprachlichen (heterogen sind) und der Wertung dienen (vgl. Burger 1998: 35; Burger 2010: 101). 
berücksichtigt und mit den behandelten Textthemen in Zusammenhang gebracht. So stellte Baldauf (1997) in ihrer empirischen Analyse von 160 Texten der seriösen Presse 100 verschiedene Metaphernkonzepte und ihnen entsprechende Metaphernsysteme fest (insgesamt 5794 metaphorische Ausdrücke, vgl. Baldauf 1997: 97). Diese 100 Metaphernkonzepte wurden auf 24 abstrakte Subkonzepte zurückgeführt, die in verschiedenen Erfahrungen rekurrent sind und als Teil eines Merkmalclusters im Sinne der revidierten Prototypentheorie ein Konzept konstituieren, z. B. das Subkonzept NEGATIV die Konzepte Krankheit, Schmerz, Dunkelheit, schlechter Geschmack. Wenn in einem konkreten Kontext ein Subkonzept aus dem das betreffende Konzept konstituierenden Merkmalcluster als relevanter Bedeutungsaspekt hervortritt, treten sprachliche Realisierungen dieser Metapher auf. Da sich ein Konzept aus einem Cluster verschiedener Subkonzepte zusammensetzt, kann die Thematisierung eines Konzepts zur Mischung verschiedener (konzeptueller) Metaphern führen, z. B. ist die Mischung von Weg- und Behälter-Metaphorik im Satz „An dieser Stelle wird die Diskussion inhaltsleer" auf die momentane Relevanz der Subkonzepte des Prozesshaften sowie der Abgrenzbarkeit der Diskussion zurückzuführen (vgl. Baldauf 1997: 261, 265-267). Die von Baldauf festgestellten Subkonzepte sind auch in den MT-Leitartikeln zu finden, was in der folgenden Übersicht anhand von MT-Beispielen belegt wird. ${ }^{9}$

Im Einzelnen sind es (1) bei den Attributsmetaphern die Subkonzepte NEGATIV IST DUNKEL (z. B. „in unserer Brust wills [...] nicht helle werden. Dort regt sich riesengroß dunkler Zweifel, es fehlt der lichte Glaube an den verheißenen Frieden“, 24.12.90, Z. 8); ${ }^{10}$ NEGATIV IST SCHWER (z. B. „die schweren Krisen“, 30.10.93, Z. 35); NEGATIV IST SCHLECHTER GESCHMACK (z. B. ,,die Bitterkeit, welche der zehnjährige Druck erzeugt hatte“, 25.11.92, Z. 93); NEGATIV IST SCHMERZHAFT (z. B. „in unser politisches Leben einen frischen Geist bringen, den wir schon lange schmerzlich vermißt haben“, 29.9.80, Z. 127); POSITIV IST HELL (z. B. „am Schlusse seiner glänzenden, geistvollen Rede“, 2.5.81, Z. 64; s. o. „der lichte Glaube“, 24.12.90, Z. 10); POSITIV IST LEICHT (z. B. „die Steuerlast zu erleichtern“, 29.9.80, Z. 99); POSITIV IST GUTER GESCHMACK (z. B. „die Süßigkeiten des Herrschens im Ministerfauteuil“, 25.1.82, Z. 44); MASSE IST PHYSISCHE KRAFT (z. B. „Allein es [= das deutsche Volk] zagt darob nicht, weil es sich stark genug fühlt auch diese Zeit kraftvoll zu überdauern“, 25.11.92, Z. 127); BEDEUTUNG IST SCHWER (nur im größeren MT-Teilkorpus z. B. „,so daß die Alttschechen sehr gewichtige Gründe haben, [...]“, 16.2.89, Z. 58); EMOTIONALITÄT IST WARM / KALT (z. B. „in der Hitze der Discussion“, 12.10.81, Z. 83; „Versicherungen ihrer Friedensliebe, [...] ihrer heißen Gefühle für die Deutschen“, 28.7.85, Z. 61; mit dem Konzept der Kälte wird z. B. Angst oder Unfreundlichkeit, aber auch das Fehlen von Emotionen konzeptualisiert, z. B. „in kühler Gleichgiltigkeit gegen die politischen Kämp-

9 Die metaphorischen Ausdrücke im $M T$ wurden nach einer ersten intuitiven Zuordnung überprüft mithilfe von Duden (2007) und ggf. weiteren im Literaturverzeichnis angeführten Wörterbüchern sowie den Auflistungen in der verwendeten Sekundärliteratur zur Metaphorik.

10 Neben dem Erscheinungsdatum des zitierten Leitartikels wird die Zeile angegeben, an der der Beleg anfängt. Da alle Texte aus dem 19. Jh. stammen, wird die Jahresangabe verkürzt. In Zitaten wird die Originalschreibweise beibehalten, die Metaphern werden hier aber kursiv markiert. 
fe“, 12.6.83, Z. 8; im größeren Teilkorpus: „mit schneidender Kälte weist die clericale Partei die ihr entgegengestreckte Hand zurück“, 15.3.87, Z. 32; vgl. Baldauf 1997: 264; 97-118).

Bei den ontologischen Metaphern (2) geht es um das Subkonzept EXISTENZ IST GEGENSTÄNDLICHKEIT (z. B. „gegen die Deutschen wurde [...] der Vorwurf der absoluten Unverträglichkeit geschleudert", 28.7.85, Z. 79; vgl. Baldauf 1997: 264; 119-122); bei den bildschematischen Metaphern (3) um die Subkonzepte BEGRENZTHEIT IST EIN BEHÄLTER (z. B. „[...] als ob eine Fraction voller Entrüstung [...] sich in die Opposition begebe“, 19.8.87, Z. 49); PROZESSHAFTIGKEIT IST EIN WEG (z. B. „wie sodann beim Scrutinium vorzugehen ist, falls die Wahl auf diesem Wege erfolgt", 30.8.92, Z. 120); POSITIV IST OBEN / NEGATIV IST UNTEN (z. B. „er hat die Sonnenhöhe seines Ruhmes überschritten und abwärts, abwärts geht es mit ihm und seinem System, dem Ende zu“, 30.10.93, Z. 16); VIEL IST HOCH / WENIG IST UNTEN (z. B. „daß die Erregung des deutschen Volkes eine nie gekannte Höhe erreicht und daß er [...] vergebens an die hohe Begabung [...] des Deutschthums in Oesterreich appelliren würde“, 25.11.92, Z. 104; „ein Gesetz, welches die bescheidenen Forderungen [...] noch tiefer herabdrückte“, 30.6.86, Z. 70); ÜBEREINSTIMMUNG / VERTRAUTHEIT IST NÄHE / NICHT-ÜBEREINSTIMMUNG IST DISTANZ (z. B. „wie Graf Taaffe [...] sich schließlich ganz und gar mit den [...] Alttschechen Eins fühlte“, 10.8.89, Z. 90; „die auch weit davon entfernt sind, sich [...] nasführen zu lassen“, 21.9.91, Z. 90); KRÄFTEVERTEILUNG IST GLEICHGEWICHT (z. B. „das Ziel [...], die verschiedenen Nationalitäten zu balanciren“, 19.8.87, Z. 109; vgl. Baldauf 1997: $265 ; 123-177)$.

Schließlich gehören zu den Konstellationsmetaphern (4) die Subkonzepte ERKENNTNIS IST (VISUELLE) WAHRNEHMUNG (z. B. - mit Metonymie kombiniert - „Ausdruck einer Lage, deren wahre Beschaffenheit keinem politischen Auge mehr verborgen ist“, 25.1.82, Z. 13); INTERESSENKONFLIKT IST HANDEL (z. B. „um welchen Preis die Verständigung zu haben wäre“, 28.7.85, Z. 66), KRIEG („ein Moment, in welchem dies- und jenseits der Leitha das Deutschthum allen möglichen Angriffen ausgesetzt ist und Feudale, Clericale und Nationale in geeinter Schlachtordnung gegen uns anrücken", 29.9.80, Z. 64), SPIEL („Sie sahen ihre Karten bald aufgedeckt“, 21.9.91, Z. 25) oder SPORT (manchmal zugleich einerseits als Spiel-, andererseits - und im MT-Kontext eher - als Kriegsmetaphern zu deuten, z. B. „Diese Gegner [...], die sich gestern eine schwere Niederlage holten“, 1.7.90, Z. 57); BEGRENZTHEIT IST WERT (z. B. Frieden ist ein wertvolles Gut: „auf ihre Kosten soll das sogenannte ewige Reich des Völkerfriedens in Oesterreich constituirt werden“, 18.8.84, Z. 21, zugleich eine Handels-Metapher); EXISTENZ (+ KAUSATION) IST PERSONIFIKATION / ANIMATION (z. B. „in uns lebte die Ueberzeugung, daß $[\ldots]$ “, 23.2.85, Z. 36; auch Pflanzen-Metaphern gehören hierher wie „auf diesen Bahnen wächst [...] nicht Frieden und Eintracht; auf ihnen blüht nur der Dornenstrauch der Sorge für die Völker Oesterreichs“, 24.12.90, Z. 113); STRUKTURIERTHEIT IST EIN BAUWERK (z. B. „welche Grundlagen für eine gesunde parlamentarische Parteibildung sind gelegt worden?", 18.8.84, Z. 51); MASSE IST WASSER / FLUT (z. B. „wer sich einbildet, eine Sonderstellung einnehmen zu können, wird von der Fluth verschlungen“, 12.6.83, Z. 60); NEGATIV IST KRANKHEIT (z. B. „Bereits hatte es den Anschein, als ob der croatische Krankheitsproce $\beta$ einen normalen Verlauf nehmen und das hart mitgenommene Land 
nun mit raschen Schritten der vollen Gesundung entgegen gehen werde“, 16.1.84, Z. 1; vgl. Baldauf 1997: 265; 178-244). Das Auftreten solcher Metaphern sei nach Baldauf weder textsorten- noch (wie Wörterbucheinträge nachgewiesen haben) an den Funktionalstil der Presse gebunden, sondern - wie schon erwähnt - nur vom jeweils thematisierten Gegenstandsbereich abhängig (vgl. Baldauf 1997: 96-97).

\subsection{Funktionen von Metaphern}

In journalistischen Texten haben die Metaphern verschiedene Funktionen. Einerseits beteiligen sie sich an der Textkohärenz - Metaphern werden häufig als intensivierte Formulierung des vorher oder nachher wörtlich gesagten verwendet (vgl. Burger 2010: 157-158). Metaphern stehen oft auch (kontaktherstellend) in den Überschriften und am Anfang eines Textes oder Textabschnittes, und wenn sie auch am Ende von Texten / Textabschnitten vorkommen, dienen sie deren Rahmung (vgl. Burger 2010: 155157). Andererseits haben sie verschiedene pragmatische Funktionen: Sie machen den Text dank Veranschaulichung verständlicher, aufgrund der Analogie zwischen einem einfachen, allgemein bekannten Bildspenderbereich (z. B. dem Kartenspiel) und einem komplexen bildempfangenden Bereich (z. B. den politisch-ökonomischen Verhandlungen hinsichtlich einer Währungskrise) kann den Lesern etwas Unbekanntes nähergebracht werden. Küster bezeichnet dies als die sozial-integrative Funktion der Metapher - sie vermittelt zwischen Bevölkerungsgruppen, die sich im bildempfangenden Bereich auskennen (dazu gehört auch der Autor des Textes), und jenen, die wenig davon verstehen (unter anderen i. d. R. auch der Leser, vgl. Baldauf 1997: 17-18, 270, 276; Kohl 2007: 67-68; Küster 1978: 98-99; Weydt 1992: 40-41). Metaphern dienen weiter der (emotionalen) Wertung, sie können die Aussage (z. B. das Hauptargument) verstärken, als ästhetischer Reiz den Text auffälliger, interessanter, unterhaltsam machen (besonders beim Spiel mit der wörtlichen und übertragenen Bedeutung des Ausdrucks), aber sie können auch als Mittel der Selbstdarstellung des Autors fungieren (vgl. Burger 1998: 32-36; Burger 2010: 100-104; Gehr 2014: 11, 20, 29-33; Kohl 2007: 64-72; Küster 1978: 76-77; Lüger 1995: 35, 38, 136; Malá 2003: 309-311).

Die durch eine Metapher evozierte anschauliche Vorstellung eines Sachverhalts kann die Rezipienten in den Glauben versetzen, den betreffenden Sachverhalt zu verstehen, was trügerisch sein kann - dem Leser kann vielleicht nur klar sein, wer oder was positiv bzw. negativ bewertet wird. Die scheinbare Anschaulichkeit kann auch dazu eingesetzt werden, die Rezipienten zu einer bestimmten Haltung in Bezug auf einen Sachverhalt und zu entsprechenden Handlungen zu stimulieren. Küster bezeichnet dies als die ideologische Funktion der Metapher, die manipulativ sein kann, Baldauf spricht von „Leitfunktion“ (vgl. Baldauf 1997: 273-275; Kohl 2007: 70-71; Küster 1978: 81-82, 99-100). Wenn z. B. Zuwanderung als Welle / Flut / Strom / Schwemme... unser Land überschwemmt, dann müssen wir uns vor diesem Naturereignis schützen - wir sollten Dämme bauen, um uns abzuschotten; so werden die Metaphern im Migrationsdiskurs fast ausschließlich gegen die Migration eingesetzt. Durch die Wahl eines Bildspenderbereichs kön- 
nen bestimmte Aspekte einer Sache hervorgehoben, andere unterdrückt werden. Aus der Verwendung bestimmter Metaphern lässt sich also auf die Argumentationsrichtung schließen (vgl. Baldauf 1997: 80, 262; Küster 1978: 70, 100; Niehr 2014: 147-148; Polenz 1991: 55).

Insgesamt ist also die Art und Weise der Metaphernverwendung in meinungsbetonten Texten abhängig nicht nur vom journalistischen Ressort (Politik, Wirtschaft, Sport, Kultur) und vom Thema des Beitrags, sondern auch von der jeweiligen Textsorte, vom Typ des Presseorgans (Qualitäts- vs. Boulevardzeitung usw.), vom Autor und auch von der für die Verfassung des Textes zur Verfügung stehenden Zeit (vgl. Gehr 2014: 12-13, 24; Malá 2009: 128-129).

\section{Metaphern im untersuchten Textkorpus}

Bei der Untersuchung der in den $M T$-Leitartikeln verwendeten Metaphern ging man zuerst von ihren Herkunftsbereichen aus. Da die Aufgabe des $M T$ die „Verteidigung“ der Vormacht der deutschen Bevölkerung in der Monarchie war, wurde als erste die zu erwartende militärische Metaphorik (die konzeptuelle Metapher POLITIK IST KRIEG / KAMPF) untersucht. Sie wurde zuerst in den Schlagzeilen gesucht, danach wurden weitere Ausdrücke aus diesem Herkunftsbereich im dazugehörigen Haupttext identifiziert und ihre Beteiligung an der Herstellung der Textkohärenz wurde beschrieben. Dabei wurde der genaueren Position der Metaphern im Text Aufmerksamkeit geschenkt - ob sie z. B. vor allem in der Texteinleitung oder im zentralen, argumentativen Teil vorkommen bzw. im ganzen Text verstreut sind. Anschließend wurden in den einzelnen Strukturteilen der übrigen Texte die Belege für diesen Herkunftsbereich aufgesucht. Da am Aufbau einzelner Texte und Textabschnitte in der Regel Metaphern aus mehreren Bereichen beteiligt sind, wurde dies als „Leitfaden“ bzw. Übergang zur Beschreibung weiterer Metaphern und Metaphernmodelle genutzt. So konnte nämlich zugleich beobachtet werden, wie der Verfasser mit Metaphern arbeitet. Zur Systematisierung wurde schließlich die Einteilung von Christa Baldauf (1997) verwendet. Einige der von ihr in 160 journalistischen Texten festgestellten 100 Metaphernkonzepte wurden in den 20 thematisch nicht so vielfältigen $M T$-Leitartikeln nicht versprachlicht. Umgekehrt wurden im $M T$ aber noch einige weitere Metaphernkonzepte realisiert bzw. einige dort festgestellte einfache / allgemeinere Metapherntypen (ontologische Metaphorik, metaphorische Animation abstrakter Konzepte) könnten komplexeren / spezifischeren, von Baldauf nicht erwähnten Metaphern zugeordnet werden (vgl. dazu Baldauf 1997: 245). Es geht um die Konstellationsmetaphern POLITIK IST HANDWERK / GEWERBE (z. B. „Die Bürger der Hauptstadt können bereits ersehen, wohin sie von jenen falschen Biedermännern geführt werden, die [...] unter dem Aushängeschild einer orthodox demokratischen Gesinnung [...] ihr verrätherisches Handwerk treiben“, 12.6.83, Z. 113; „Wenn Meister der Staatskunst über solche Haltung ein Zeugniß ausstellen sollten, könnten sie dem herrschenden Systeme die Befähigung zur Leitung eines großen mächtigen Staatswesens ertheilen?“, 19.12.82, Z. 75); POLITIK IST LANDARBEIT (z. B. „Die Herren 
Mandl und Lueger [...] sehen die Früchte ihrer Politik in erschreckender Geschwindigkeit heranreifen“, 12.6.83, Z. 35; „[...] Graf Taaffe selbst würde über dem Versuche es dennoch zu thun seine gute Laune verlieren, ohne staatsmännischen Ruhm zu ernten“, 25.11.92, Z. 138); POLITIK IST ESSEN (z. B. „Proßnitz dagegen soll mit Wischau [...], Austerlitz und Butschovitz zusammengekoppelt werden, deren Vertreter selbstverständlich dann ein Mann nach dem Geschmacke der Herren Schrom und Fanderlik wäre“, 23.2.85, Z. 57); POLITIK IST RELIGION (z. B. „Der patriotische Gedanke der Staatseinheit habe keine treueren Priester als die Deutschen in Oesterreich“, 12.10.81, Z. 94) ${ }^{11}$ oder POLITIK IST MUSIKSPIELEN (z. B. „So lange nicht die unerbittliche Nothwendigkeit eine Aenderung des herrschenden Systems gebietet, wird auf denselben Instrumenten weiter gespielt werden, wenn auch mit einigen Aenderungen im Text oder an der Melodie“, 30.6.86, Z. 52). ${ }^{12}$

Nicht alle im MT identifizierten Alltagsmetaphern sind stilistisch relevant. Zu den auffallenden Metaphern gehören jedenfalls die Konstellationsmetaphern für die politische Tätigkeit - neben den gerade erwähnten noch die Metaphernmodelle POLITIK IST THEATER, SPIEL, HANDEL, SCHIFFFAHRT bzw. allgemeiner REISE, KRIEG / KAMPF. Im Folgenden werden als Beispiel die Realisierungen des zuletzt genannten Metaphernmodells näher vorgestellt.

\subsection{Die Kampfmetaphorik in den MT-Leitartikeln}

Im betrachtenden Leitartikel mit der Überschrift „Die Frontänderung der Tschechen“ (MT 19.8.87) bezieht sich die militärische Metapher Frontänderung auf die politische Situation in Böhmen und im österreichischen Parlament: die Parteien der Alt- und der Jungtschechen kämpfen gegenwärtig nicht mehr gegeneinander, sondern „,bekämpfen“ (Z. 3) gemeinsam die Regierung, konkret den Unterrichtsminister Gautsch und das von ihm erlassene Regulativ für die Mittelschulen (vgl. MT 3.8.87, S. 6; 4.8.87, S. 2-3, 5.8.87, S. 5-6; 10.8.87, S. 2, 5, 7), das u. a. „Aufhebung etwelcher tschechischer Gymnasien“ (19.8.87, Z. 128 des Leitartikels) zur Folge hätte. Die Kampfmetapher wird in der Texteinleitung ausgeführt:

„Nachdem die Jungstschechen Monate lang einen hartnäckigen Feldzug gegen ihre älteren Genossen geführt haben, weil diese nicht entschieden genug die Rechte der großmächtigen Nation

11 Manche MT-Belege für diese Metapher können allerdings auch auf die Bibel zurückgeführt werden, sind also auch intertextuell zu deuten, vgl. „Tag für Tag tönen weise Mahnungen an unser Ohr doch ja der Regierung Taaffe zu vertrauen [...]. Die Propheten, die so prophezeihen, finden aber beim deutschen Volke in Oesterreich keinen Glauben und je eifriger sie ihre Weissagungen ertönen lassen, desto weniger glaubt das Volk an sie“ (29.9.80, Z. 1).

12 Einige Musikmetaphern könnten der konzeptuellen Metapher POLITIK IST THEATER zugeordnet werden, wenn Theater im weiteren Sinne verstanden wird und auch Musikaufführungen wie Opern einschließt. Im $M T$ werden aber noch weitere Musikarten metaphorisch verwendet, z. B. im Leitartikel „Die Heilbotschaft“ (18.12.89) wird in Übereinstimmung mit der in der Überschrift versprachlichten Metapher POLITIK IST RELIGION die Texteinleitung mit der Metapher „es wird auf der officiösen Orgel wieder das alte Lied ertönen“ (Z. 19) abgeschlossen. 
gegen die Wiener Regierung verfochten, stehen jetzt alle Armeecorps des früher uneinigen Heeres in voller Rüstung da, die Waffen gegen das Ministerium gerichtet.“ (Z. 4-11)

Das Bild der Alttschechen als künftige „Leibgarde“ des Ministeriums (Z. 15) löste sich also auf. Es wird zwar die Möglichkeit erwogen, dass „Herr von Gautsch als Opfer“ dieses Kampfes „fallen gelassen“ werden könnte (Z. 34-35), aber auch, dass die Regierung und konkret der Ministerpräsident Taaffe den Forderungen der Tschechen diesmal nicht nachgibt. Diese zwei Möglichkeiten und auch ihre Folgen für die liberale Opposition werden im zentralen Teil diskutiert. Für die erste Möglichkeit sprächen die bisherigen Erfahrungen mit dem Ministerium Taaffe, das schon oft „von Position zu Position [zurückJwich“ (Z. 54). ${ }^{13}$ Deshalb könnte ,jedes voreilige Triumphgeschrei der Opposition“ (Z. 70-71) ein politischer Fehler sein. Der Autor des Leitartikels unterstützt die zweite Möglichkeit, was schon in der Charakteristik der politischen Situation am Schluss der Texteinleitung mithilfe von negativ konnotierten stilistischen Elementen deutlich wird (einer Hyperbel und zwei verschränkten bildschematischen Metaphern - VORGEHENSWEISE IST EIN WEG und POSITIV IST OBEN / NEGATIV IST UNTEN bzw. genauer VERBESSERUNG IST AUFWÄRTSBEWEGUNG / VERSCHLECHTERUNG IST ABWÄRTSBEWEGUNG): Graf Taaffe scheine einen Versuch zu machen „sich von dem allerärgsten Terrorismus der tschechischen Fraction zu befreien“ und „auf dem gefährlichen Rutschterrain [...], welches vor acht Jahren betreten wurde“ Halt zu machen (Z. 36-41). Dies wird mit einem modifizierten geflügelten Wort positiv gedeutet, da er so „dem Staate gibt, was des Staates ist“ (Z. 39). ${ }^{14}$ Die Unterstützung dieser Möglichkeit wird im zentralen Teil (unter Anwendung von Kampf-, aber auch einer der erwähnten bildschematischen Metaphern) bekräftigt, indem der Autor indirekt darauf hinweist, dass man „Dasjenige, was bereits von der Krone sanctioniert ist, nicht dem Ansturme einer einzelnen Fraction preisgeben“ sollte (Z. 75-77); indem er dem Ministerium Taaffe einredet, dass es auch ohne Unterstützung von Tschechen regierungsfähig bleibe (dass dadurch nicht sein „Fall [...] herbeigeführt werden muss“, Z. 78-80); indem er die aktuelle Situation mit früheren Situationen vergleicht und die Möglichkeit, dass „es zu keinem Friedensschlusse zwischen den Tschechen und dem Grafen Taaffe kommt“ (Z. 94-96), zu verharmlosen sucht („Sehr wahrscheinlich wird eben ein etwaiger Ansturm der Tschechen gegen das Ministerium Taaffe-Gautsch gerade so wenig von Erfolg begleitet sein, als es der Ansturm der Deutschen war“, Z. 90-94). Mit (in diesem Kontext spöttisch klingender) Berufung auf das Regierungsprogramm der Versöhnung der Nationalitäten („das Ziel [...], die verschiedenen Nationalitäten zu balanciren“, Z. 109-111, das GLEICHGEWICHT-Schema) versucht der Autor schließlich Graf Taaffe herauszufordern, indem er auf seine Ehre

13 Die sich wiederholende „Frontänderung“ wird deshalb auch als „Spiel“ bezeichnet (Z. 45). Eine andere in der Texteinleitung für die Veränderlichkeit des politischen Geschehens verwendete SPIEL-Metapher ist das oft als Spielzeug gebrauchte Kaleidoskop: „Das war ein Augenblicksbild; aber in unserem politischen Kaleidoscope wechseln die Dinge sehr häufig, und so hat sich der Prospect auch jetzt wieder verschoben“ (19.8.87, Z. 18-21).

14 Vgl. dem Kaiser geben, was des Kaisers ist ,seine Pflicht gegenüber der Obrigkeit erfüllen` (nach Matth. 22, 21; DU). 
appelliert - er habe „eine Möglichkeit vor sich, die ein Staatsmann von Kühnheit ${ }^{15}$ sicher ergreifen würde“ (Z. 114-115). Der Autor gibt zwar gleich (wieder mit einer WEG-Metapher) zu, dass es nicht seine Aufgabe sei, dem Grafen „neue Wege zu weisen“, Z. 116-117), darauf folgt aber - wie es der Struktur eines Leitartikels entspricht - die Bestätigung der zentralen Meinungsäußerung, dass Graf Taaffe, wenn er den Tschechen in der Frage des betreffenden Erlasses nicht nachgibt und ihre Unterstützung verliert, nicht das Ende seiner Ministerpräsidentschaft befürchten müsse. In der Rechtfertigung wird - als Schließung des am Textanfang eröffneten Rahmens - noch eine Kampfmetapher verwendet, wenn diejenigen genannt werden, die „den Erlaß des Herrn von Gautsch gegen die Angriffe der Tschechen vertheidigt" haben (Z. 124-125).

In der Überschrift wurden sonst Kriegsmetaphern erst in 13 Leitartikeln des größeren Teil-Korpus von 163 Texten festgestellt. Nur das Gegenteil von Krieg, Frieden (was allerdings Bestandteil desselben Szenarios ist), kommt in der Überschrift des Weihnachtsleitartikels vom 24.12.1890 vor und wird auch in allen Textstrukturteilen wiederaufgenommen. Ähnlich wie im oben vorgestellten Leitartikel zieht sich noch die Kriegsmetaphorik durch den ganzen Haupttext des Leitartikels „Die Städtewahlen in Mähren“ (1.7.90). An die Texteröffnung „Die Städte Mährens standen gestern im Feuer des Wahlkampfes [...]“ (Z. 1-2 $\mathrm{Ge}^{16}$ ) folgt die Erwähnung einiger „Niederlagen“ (Z.18, II.E; Z. 25 Ge, III.A), denen im argumentativen Teil Siege entgegengesetzt werden: „das Deutschthum [bestand] fest und machtvoll den Kampf“ (Z. 30-31), „[behauptete] selbst dort, wo der gegnerische Ansturm bis zum Straßenexcesse ausartete, unverzagt und ungebrochen den Kampfplatz" (Z. 32-34), drei Mandate wurden „zurückerobert“ (Z. 35), der „Sieg in Proßnitz“ wird als ruhmvoll bezeichnet, ,weil an keinem anderen Puncte der Wahlschlacht der Kampf ein so heißer war, weil nirgends die Kämpfer so ausdauernd ihre Pflicht erfüllten“ (Z. 39-43), wobei es hauptsächlich „die Deutschliberalen und die Juden [...] sind, die so opfervollen Kampf ausfochten und dem Deutschthume eine feste Bastion zurückeroberten" (Z. 53-57), „einen herrlichen Wahlsieg [errangen]“ (Z. 62) bzw. - wie es im Schlussteil nochmals betont wird - „im harten Ringen nicht nur ihre meisten Positionen behaupteten, sondern auch früher verlorene Positionen zurückeroberten“ (Z. 82-84 Ge, V.S). Die „Gegner in den eigenen Reihen des Deutschthums“ holten sich dagegen in Schlesien „eine schwere Niederlage“, aber sein Gebiet soll nicht „in einen Kampfboden verwandelt“ werden (Z. 73-76, III.A), der Autor hofft, dass „die schlesischen Städte die [...] Niederlage der Landgemeinden wieder wettmachen und sich ebenbürtig den Kämpfern in den deutschmährischen Städten erweisen“ (Z. 78-81 Ge, V.S). Die Deutschliberalen mahnt er im Schlussteil:

„Es gilt nur, sich an dem erfochtenen Wahlsiege nicht zu berauschen; es heißt vorwärts streben und sich nicht [...] von den angriffslüsternen Gegnern in die Defensive drängen lassen. Wir müssen endlich wieder die Offensive ergreifen und zurückzugewinnen trachten [...], was wir [...] vor sechs Jahren, als die Gegner unter der offenen Patronanz des Grafen Schönborn kämpften, verloren.

15 Vgl. kühn [mhd. küene, ahd. kuoni = mutig, stark; im Kampf erfahren, tüchtig;,..] (DU).

16 Zu Abkürzungen Ü, E, A, S für Textabschnitte s.o. Kap.1. Die Verwendung der Metaphern zur Gliederung des Textes wird mit Ge (bei der Einleitung eines Abschnitts) bzw. Gs (beim Abschluss) markiert. 
[...] die Aera der Kämpfe um jeden Fleck deutschen Bodens [ist] noch nicht abgeschlossen [...]“ (Z. 84-98, V.S).

Insgesamt wurden in den 20 Leitartikeln 210 metaphorische Ausdrücke (Ein- und Mehrwortmetaphern) aus dem Herkunftsbereich Krieg / Kampf (einschließlich des Gegenteils - des Friedens), d. h. Versprachlichungen der konzeptuellen Metapher POLITIK IST KRIEG / KAMPF, festgestellt. ${ }^{17}$ Sie werden im Folgenden in alphabetischer Reihenfolge mit teilweiser Berücksichtigung von Wortbildungsnestern angeführt. Obwohl auch die Beschreibung ihrer Einbindung in den Kontext interessant wäre (z. B. wer wen bzw. was bekämpft), wird hier aus Platzgründen darauf verzichtet.

Abwehr (29.9.80, Z. 10, II.E; Z. 120, III.A), abwehren (12.6.83, Z. 64, III.A; Z. 75, IV.WG, 30.6.86, Z. 76, III.A); Angegriffene (18.8.84, Z. 20, II.E), Angreifer (18.8.84, Z. 31 Gs, II.E), Angriffe (29.9.80, Z. 66, III.A; 19.8.87, Z. 124, V.S) / Sturmangriff (18.8.84, Z. 62, III.A), angriffslüstern (1.7.90, Z. 87, V.S); gegen jmdn. anrücken (29.9.80, Z. 68, III.A); Ansturm (19.8.87, Z. 76, 91, 93, III.A; 1.7.90, Z. 32, III.A), anstürmen (29.4.93, Z. 94, III.A); Armeecorps (19.8.87, Z. 9, II.E); das Banner der Völkerversöhnung (30.6.86, Z. 5, II.E); Bastion (1.7.90, Z. 56, III.A); jmdn. befehligen (10.8.89, Z. 92, III.A); Corpscommandant (25.1.82, Z. 67, III.A), Höchstcommandirender (25.1.82, Z. 67, III.A); Defensive (1.7.90, Z. 88, V.S); sich jmds. erwehren (18.8.84, Z. 53, III.A); die nationale Fahne hoch halten (12.6.83, Z. 107 Gs, III.A) / die Fahne des Fortschritts hochhalten (12.6.83, Z. 132 Gs, V.S); den Erfolg an seine Fahnen fesseln (30.10.93, Z. 7, II.E); etw. steht auf jmds. Fahne (30.10.93, Z. 49, III.A); unter den bunten Provinzialfähnchen dahinrasen (12.10.81, Z. 72, III.A); Fähnlein (29.4.93, Z. 135, III.A); die Reichsflagge entfalten (12.10.81, Z. 70, III.A); fechten in etw. ausfechten (1.7.90, Z. 55, III.A), etw. erfechten (1.7.90, Z. 85, V.S), etw.(gegen jmdn.) verfechten (19.8.87, Z. 8, II.E; 24.12.90, Z. 21, II.E); Zeitungsfehde (18.8.84, Z. 2 Ge, II.E); Feld in Feldherr (30.10.93, Z. 6, II.E), Feldzeichen (12.6.83, Z. 56, III.A), Feldzug gegen jmdn. führen (19.8.87, Z. 5, II.E), Ausgleichsfeldzug (29.4.93, Z. 82, III.A); im Feuer des Wahlkampfes stehen (1.7. 90, Z. 1 Ge, II.E); jener / der parlamentarische / nationale Flügel (29.9.80, Z. 86; IV.WG; 30.6.86, Z. 75, III.A); Frieden (24.12.90, Z. 11, II.E) / Völkerfrieden (18.8.84, Z. 20, II.E; 24.12.90, Z. 36, II.E), intertextuell motiviert: „Frieden auf Erden“ (24.12.90, Z. 6, II.E; Z. 108, IV.G), Frieden und Eintracht (24.12. 90, I.Ü; Z. 115, 117, 122 Gs, III.A; Z. 138 Gs, V.S), Frieden und Versöhnung (29.9.80, Z. 10, II.E), Friedensliebe (28.7.85, Z. 62, III.A), Friedensschluss (19.8.87, Z. 94, III.A), Friedensvorschläge machen (28.7.85, Z. 35, III.A), Friedenswerk (24.12.90, Z. 43, III.A), friedlich (16.1.84, Z. 59, III.A; 28.7.85, Z. 89, 91, III.A; 1.7.90, Z. 73, IV.WG), friedfertig (18.8.84, Z. 25, II.E); Frontänderung (19.8.87, I.Ü); sich (unversöhnt) gegenüberstehen (2.5.81, Z. 7 Ge, 24, III.A; 18.8.84, Z. 42, III.A) / einer Sache gegenüberstehen (21.9.91, Z. 49, III.A), auf [irgend]welcher Seite stehen (12.6.83, Z. 86, III.A); Gegner (20 Belege: 2.5.81, Z. 64, III.A; 12.10.81, Z. 75 Gs, III.A; Z. 80, V.S; 12.6.83, Z. 101, III.A; Z. 125, V.S; 18.8.84, Z. 24, II.E; 23.2.85, Z. 37, III.A; 28.7.85, Z. 75, III.A; 10.8.89, Z. 142, III.A; 1.7.90, Z. 6, II.E; Z. 51, 57, 64, III.A; Z. 88, 92, V.S; 24.12.90, Z. 42 Ge, III.A; 25.11.92, Z. 45, III.A; 29.4.93, Z. 58, 138, III.A; 30.10.93, Z. 8, II.E), eine unversöhnliche Gegnerschaft (12.6.83, Z. 44, III.A), gegnerisch (1.7.90, Z. 32, III.A); der Generalstab (29.9.80, Z. 133, III.A);

17 Aufgrund des Kriegsszenarios wurden auch einige mehrdeutige Ausdrücke dieser Konstellationsmetapher zugeordnet (z. B. Gegner, jmdm. gegenüberstehen, Position, Opfer, verlieren). 
„Gewehr bei Fuß“ (25.11.92, Z. 69, III.A); Heer (19.8.87, Z. 9, II.E), Heeresfolge (30.6.86, Z. 91, V.S) / Heerfolge (29.4.93, Z. 56, III.A), Heerschaar (16.1.84, Z. 53, III.A); Heldenthat (16.1.84, Z. 82, IV.G); der / jmds. Kampf (um etw. / gegen jmdn. / zwischen jmdm. u. jmdm.) (17 Belege: 29.9.80, Z. 120, III.A; 12.10.81, Z. 49, III.A; 25.1.82, Z. 108, III.A; 19.12.82, Z. 99, V.S; 12.6.83, Z. 9, II.E; Z. 52, 57, III.A; 16.1.84, Z. 95 Gs, III.A; 18.8.84, Z. 57, 60, 84, III.A; 1.7.90, Z. 31, 42, 55, III.A; Z. 97, V.S; 29.4.93, Z. 80, III.A; 30.10.93, Z. 74, III.A), den Bruderkampf führen (16.1.84, Z. 83, IV.WG), Nationalitätenkampf (12.10.81, Z. 65, IV.WG), Wahlkampf (1.7.90, Z. 1, II.E), Kampfmuth (1.7.90, Z. 4, II.E), Kampfboden (1.7.90, Z. 75 Gs, III.A), Kampfplatz (1.7.90, Z. 34, III.A), (für etw.) kämpfen (12.6.83, Z. 60, III.A; 16.1.84, Z. 49 Ge, III.A; 1.7.90, Z. 93, V.S); jmdn. / etw. bekämpfen (19.8.87, Z. 3 Ge, II.E; 10.8.89, Z. 12, 140, III.A; 25.11.92, Z. 84, III.A; 29.4.93, Z. 138, 138, III.A), Kämpfer (1.7.90, Z. 43, III.A; Z. 80 Ge, V.S); Kriegserklärung (29.9.80, Z. 75, III.A); Linie (19.8.87, Z. 60, III.A), in erster Linie (12.10.81, Z. 67, IV.WG); Lager (12.10.81, Z. 10, II.E; Z. 79 Ge, V.S; 12.6.83, Z. 73, III.A; 16.1.84, Z. 53, III.A; 30.10.93, Z. 8, II.E), Regierungslager (25.11.92, Z. 75, III.A); Leibgarde (19.8.87, Z. 15, II.E); Macht ${ }^{18}$ in Hauptmacht (29.4.93, Z. 134, 137, III.A); Niederlage (12.6.83, Z. 93, III.A; 10.8.89, Z. 99, III.A; 1.7.90, Z. 18, II.E; Z. 25 Ge, 59, III.A; Z. 79 Ge, V.S); jmdn. niederschmettern (28.7.85, Z. 39, III.A); Offensive (1.7.90, Z. 89, V.S); Opfer (18.8.84, Z. 20, II.E; 19.8.87, Z. 34, II.E), opfervoll (1.7.90, Z. 47, 55, III.A); Ordre pariren ${ }^{19}$ (30.6.86, Z. 90, V.S); Positionen behaupten / zurückerobern (1.7.90, Z. 82 Ge, 83 Ge, V.S) / von Position zu Position zurückweichen (19.8.87, Z. 54, III.A); (in) seine(n) (eigenen) Reihen (19.8.87, Z. 48, 62, III.A; 1.7.90, Z. 45, 51, 57, III.A; 25.11.92, Z. 145, V.S); sich aus etw. rekrutiren (29.9.80, Z. 133, III.A); um etw. ringen (1.7.90, Z. 101, V.S), etw. erringen (1.7.90, Z. 62, III.A), das Ringen (1.7.90, Z. 82 Ge, V.S); Rückzug aus etw. (10.8.89, Z. 172, V.S); sich zu etw. rüsten (18.8.84, Z. 61, III.A) / sich zu etw. rüsten und wappnen (29.9.80, Z. 10, II.E), in voller Rüstung (19.8.87, Z. 10, II.E); schießen nur in einer intertextuell motivierten Metapher: „das Dichterwort: Gestern noch auf stolzen Rossen, / Heute durch die Brust geschossen“ (30.10.93, Z. 24 Gs, II.E); Schlacht in Wahlschlacht (1.7.90, Z. 41, III.A), Redeschlacht (2.5.81, Z. 42, III.A), Schlachtengewühl (12.10.81, Z. 69, III.A), Schlachtgeschrei (12.10.81, Z. 1 Ge, II.E), Schlachtordnung (29.9.80, Z. 68, III.A); Schlag (28.7.85, Z. 39, III.A); Sieg (18.8.84, Z. 28, II.E; Z. 45, III.A; 1.7.90, Z. 39, 64, III.A) / Wahlsieg (1.7.90, Z. 62, III.A; Z. 67, IV.G; Z. 85, V.S), siegen (1.7.90, Z. 39, III.A), sieghaft (30.10.93, Z. 5, II.E); Soldschreiber (25.1.82, Z. 128, V.S); Standarte (12.10.81, Z. 70, III.A); die Streitaxt begraben ${ }^{20}$ (24.12.90, Z. 28, II.E); die Taktik gegenüber jmdm. (30.6.86, Z. 39, III.A), tactisch (30.6.86, Z. 66, III.A); Terrorismus (23.2.85, Z.92 Gs, III.A; 19.8.87, Z.37, II.E); Triumph (29.4.93, Z. 97, III.A), Triumphgeschrei (19.8.87, Z. 71, III.A), Triumphator (29.4.93, Z. 100, III.A); Ueberläufer (16.1.84, Z. 53, III.A); die Waffen gegen jmdn. richten (19.8.87, Z. 10, II.E); sich wappnen (s. o. rüsten); Werberede (25.11.92, Z. 77, III.A); etw. gegen etw. vertheidigen (19.8.87, Z. 125, V.S), die Vertheidigung einer Sache (29.9.80, Z. 160, V.S); verlieren (25.11.92, Z. 130 Gs, III.A); kein Wallenstein ${ }^{21}$ (21.9.91, Z. 23, III.A); etw. zurückerobern (1.7.90, Z. 35, 56, III.A; Z. 84, V.S - s.o. Positionen).

\footnotetext{
18 Vgl. Macht 5. (veraltend), Heer, Truppen': mit bewaffneter M. anrücken, angreifen (DU).
}

19 Vgl. ordre parieren / einem ordre parieren, etwas befohlenes thun' (vgl. DW Bd. 13, Sp. 1461); parieren , ohne Widerspruch gehorchen'; Ordre, frz. Form von Order 1. ,[militärischer, dienstlicher] Befehl; Anweisung' (DU).

20 Vgl. Streitaxt (früher), als Hieb- od. Wurfwaffe verwendete Axt' (DU); die Streitaxt begraben, frieden schlieszen * (vgl. DW Bd. 19, Sp. 1338-1339) / ,einen Streit beenden' DU).

21 Wallenstein (1583-1634) war ein böhmischer Feldherr und Politiker (vgl. URL1). Zu Eigennamenmetaphern vgl. Thurmair (2002). 


\begin{tabular}{|c|c|c|c|c|c|c|c|}
\hline & MT & I.Ü & II.E & III.A & IV.G+WG & V.S & Insgesamt \\
\hline 1. & 29.9 .1880 & & 3 & 8 & $0+1$ & 1 & 13 \\
\hline 2. & 2.5 .1881 & & & $4(1 \mathrm{Ge})$ & & & 4 \\
\hline 3. & 12.10 .1881 & & $2(1 \mathrm{Ge})$ & $6(1 \mathrm{Gs})$ & $0+2$ & $2(1 \mathrm{Ge})$ & 12 \\
\hline 4. & 25.1 .1882 & & & 3 & & 1 & 4 \\
\hline 5. & 19.12.1882 & & & & & 1 & 1 \\
\hline 6. & 12.6.1883 & & 1 & 11 (1Gs) & $0+1$ & 2 (1Gs) & 15 \\
\hline 7. & 16.1.1884 & & & $6(1 \mathrm{Ge}, 1 \mathrm{Gs})$ & $1+1$ & & 8 \\
\hline 8. & 18.8 .1884 & & $8(1 \mathrm{Ge}, 1 \mathrm{Gs})$ & 8 & & & 16 \\
\hline 9. & 23.2 .1885 & & & 2 (1Gs) & & & 2 \\
\hline 10. & 28.7 .1885 & & & 7 & & & 7 \\
\hline 11. & 30.6 .1886 & & 1 & 4 & & 2 & 7 \\
\hline 12. & 19.8.1887 & 1 & $10(1 \mathrm{Ge})$ & 9 & & 2 & 22 \\
\hline 13. & 10.8.1889 & & & 5 & & 1 & 6 \\
\hline 14. & 1.7.1890 & & $5(1 \mathrm{Ge})$ & $28(1 \mathrm{Ge}, 1 \mathrm{Gs})$ & $1+1$ & $15(5 \mathrm{Ge})$ & 50 \\
\hline 15. & 24.12 .1890 & 1 & 5 & 5 (1Ge, 1Gs) & $1+0$ & 1 (Gs) & 13 \\
\hline 16. & 21.9 .1891 & & & 2 & & & 2 \\
\hline 17. & 30.8 .1892 & & & & & & 0 \\
\hline 18. & 25.11 .1892 & & & 6 (1Gs) & & 1 & 7 \\
\hline 19. & 29.4 .1893 & & & 13 & & & 13 \\
\hline 20. & 30.10 .1893 & & 6 (1Gs) & 2 & & & 8 \\
\hline $\begin{array}{l}\text { Insge- } \\
\text { samt }\end{array}$ & & 2 & $\begin{array}{c}41 \\
(4 \mathrm{Ge}, 2 \mathrm{Gs})\end{array}$ & $\begin{array}{c}129 \\
(4 \mathrm{Ge}, 7 \mathrm{Gs})\end{array}$ & $\begin{array}{c}9 \\
(3+6)\end{array}$ & $\begin{array}{c}29 \\
(6 \mathrm{Ge}, 2 \mathrm{Gs})\end{array}$ & $\begin{array}{c}210 \\
(14 \mathrm{Ge}, 11 \mathrm{Gs})\end{array}$ \\
\hline
\end{tabular}

Tab. 1 Distribution der Kriegs- / Kampfmetaphern in den MT-Leitartikeln

Wie aus der Tabelle 1 hervorgeht, kommen die meisten Kriegsmetaphern im argumentativen Teil der Leitartikel vor, am zweithäufigsten sind sie in der Texteinleitung zu finden und der dritte bevorzugte Textabschnitt ihrer Verwendung ist der Textschluss. ${ }^{22}$ Dabei werden sie 14-mal zur Einleitung und 11-mal zum Abschluss der einzelnen Strukturteile verwendet, am häufigsten (7-mal) zum Abschluss des argumentativen Teils. Fünfmal weist nur der argumentative Teil diese Metaphern auf, einmal nur der Schlusssteil. Am häufigsten kommen die Kriegsmetaphern in dem oben besprochenen Leitartikel vom 1.7.1890 vor, der die Wahlergebnisse bewertet. Keine Kriegsmetapher wurde im unterrichtenden Leitartikel vom 30.8.1892 verwendet, der die Prozedur der Wahl des Erzbischofs von Olmütz erklärt und am Ende den Wunsch ausdrückt, dass die berechtigten Wähler sich bei der bevorstehenden Wahl nicht von „politischen oder nationalen Motiven“ (Z. 193) leiten lassen.

\section{Schluss}

In diesem Beitrag wurde ein Überblick über die Art der in den Leitartikeln des Mährischen Tagblatts verwendeten Metaphern geboten. Dazu wurde von den in den Abschnitten 2 und

22 Genauer müssten die Zahlen mit der Gesamtlänge der einzelnen Abschnitte in Verbindung gebracht werden. 
3 des Beitrags vorgestellten Beschreibungsmöglichkeiten Christa Baldaufs kognitivbasierte Klassifikation der Metaphern verwendet, deren Grundlage die Art und Struktur der Herkunftsbereiche und die verschiedene Metaphernkonzepte konstituierenden Subkonzepte bilden und in welche die anderen Beschreibungsansätze integrierbar sind.

Anschließend wurde als Beispiel die aufgrund der Zielsetzung der betreffenden Zeitung und der Textsorte am ehesten zu erwartende militärische Metaphorik in den ausgewählten Texten näher dargestellt. Dabei wurden nicht nur die verschiedenen Versprachlichungen eines der Metaphernmodelle für die Politik, des Modells POLITIK IST KRIEG / KAMPF, aufgelistet, sondern es wurde auch ihre Distribution in den für einen Leitartikel charakteristischen Textteilen untersucht. An zwei Leitartikeln, in denen die Kriegsmetaphern in besonderer Häufung vorkommen, wurde die Beteiligung dieser Metaphern an der Herstellung der Textkohärenz beschrieben. Im Zusammenhang damit wurden einige weitere am Aufbau einzelner Textabschnitte beteiligte Metaphern aus anderen Herkunftsbereichen erwähnt. Sehr zahlreich sind in den MT-Leitartikeln z. B. Weg- und Bewegungsmetaphern, zu deren spezifischen Ausprägungen das Metaphernmodell POLITIK IST SCHIFFFAHRT gehört. Neben diesen wäre weiter auch die Verwendung der Theater-Metaphorik in den MT-Leitartikeln untersuchungswert, da sie aufgrund der Rolle des Theaters (und der Oper) in Olmützer Kulturleben für die Gestaltung der Schreiber-Leser-Beziehung wichtig gewesen sein dürfte.

\section{Quellen}

Mährisches Tagblatt. In: ANNO. Historische österreichische Zeitungen und Zeitschriften. http:// anno.onb.ac.at/cgi-content/anno?aid=mtb (28.6.2019).

\section{Literatur}

Baldauf, Christa (1997). Metapher und Kognition. Grundlagen einer neuen Theorie der Alltagsmetapher. Frankfurt a. M.: Lang.

Brinker, Klaus (1997): Linguistische Textanalyse. Eine Einführung in die Grundbegriffe und Methoden (4., durchges. u. erw. Aufl.). Berlin: Schmidt.

Burger, Harald (1998): Idiom and Metaphor. Their Relation in Theory and Text. In: Ďurčo, Peter (Hrsg.): EUROPHRAS 97. Phraseology and Paremiology. Bratislava: Akadémia Policajného zboru, S. 30-36.

Burger, Harald (2005): Mediensprache. Eine Einführung in Sprache und Kommunikationsformen der Massenmedien. Mit einem Beitrag von Martin Luginbühl (3., völlig neu bearb. Aufl.). Berlin: de Gruyter.

Burger, Harald (2010): Phraseologie: eine Einführung am Beispiel des Deutschen (4., neu bearb. Aufl.). Berlin: Schmidt.

Dobrovol'skij, Dmitrij - Piirainen, Elisabeth (2009): Zur Theorie der Phraseologie. Kognitive und kulturelle Aspekte. Tübingen: Stauffenburg.

Dovifat, Emil (1967): Zeitungslehre. Bd. 1 (5., neu bearb. Aufl.). Berlin: de Gruyter. 
DU = Dudenredaktion (Hrsg.) (2007): Duden Deutsches Universalwörterbuch (6., überarb. u. erw. Aufl.). Mannheim: Dudenverlag. (CD-ROM)

Duhme, Michael (1991): Phraseologie der deutschen Wirtschaftssprache. Eine empirische Untersuchung zur Verwendung von Phraseologismen in journalistischen Fachtexten. Essen: Die blaue Eule.

DW $=$ Deutsches Wörterbuch von Jacob Grimm und Wilhelm Grimm [online]. http://dwb. uni-trier.de/de/ (3.7.2019).

D11 = Dudenredaktion (Hrsg.) (2008): Duden. Redewendungen. Wörterbuch der deutschen Idiomatik (3. überarb. u. aktual. Aufl.). Mannheim: Dudenverlag.

Fedina, Martin (2011): Metafory v politickém diskurzu Václava Klause. [Metaphern im politischen Diskurs von Václav Klaus.] In: E-polis.cz, 19.10.2011. http://www.e-polis.cz/clanek/ metafory-v-politickem-diskurzu-vaclava-klause.html (1.7.2019).

Fleischer, Wolfgang (1997): Phraseologie der deutschen Gegenwartssprache (2., durchgeseh. u. erg. Aufl.). Tübingen: Niemeyer.

Föhner, Josef (1930): Olmützer Erinnerungen. Olmütz: Mährisches Tagblatt.

Gehr, Martin (2014): Metaphern und Redewendungen im politischen Kommentar. Wiesbaden: Springer VS.

Hartmann, Dietrich (1999): Zur Phraseologiebildung mittels metonymischer Prozesse aus der Sicht der kognitiven Linguistik. In: Baur, Rupprecht S. - Chlosta, Christoph - Piirainen, Elisabeth (Hrsg.): Wörter in Bildern - Bilder in Wörtern. Beiträge zur Phraseologie und Sprichwortforschung aus dem Westfälischen Arbeitskreis. Baltmannsweiler: Schneider-Verlag Hohengehren, S. 219-238.

Hegedüs-Lambert, Claudia (2010): Verfestigte Körpermetaphern und -metonymien in Idiomen mit der Nominalkonstituente Hand. In: Revue Interdisciplinaire „Textes \& contextes“ [online], 5 „Stéréotypes en langue et en discours“. https://revuesshs.u-bourgogne.fr/textes\&contextes/ document.php?id=1146 (28.6.2019).

Höppnerová, Věra (2006): Bildhaftes Wirtschaftsdeutsch. Auf Spurensuche im Metaphernbereich. In: Deutsch als Fremdsprache 43, 4, S. 233-238.

Kaňovská, Michaela (2015): Phraseme als Mittel der Rahmung in den Leitartikeln des Mährischen Tagblatts. In: Kusová, Jana - Malechová, Magdalena - Vodrážková, Lenka (Hrsg.): Deutsch ohne Grenzen. Linguistik. Brno: Tribun EU, S. 103-125.

Keller-Bauer, Friedrich (1983): Metaphorische Präzedenzen. In: Sprache und Literatur in Wissenschaft und Unterricht 51, S. 46-60.

Kispál, Tamás (2013): Methodenkombination in der Metaphernforschung. Metaphorische Idiome des Lebens. Frankfurt a. M.: Lang.

Klenovský, Jaroslav - Papoušek, Miroslav (1997-1998): Židovská obec v Olomouci - historie, osobnosti, památky. [Jüdische Gemeinde in Olmütz - Geschichte, Persönlichkeiten, Denkmäler] Olomouc: Židovská obec v Olomouci.

Kövecses, Zoltán - Szabó, Péter (1996): Idioms - A View from Cognitive Semantics. In: Applied Linguistics 17, 3, S. 326-355. https://www.researchgate.net/publication/308780565_ Idioms_A_View_from_Cognitive_Semantics (28.6.2019).

Kohl, Katrin (2007): Metapher. Stuttgart: Metzler.

Kudla, Martin (2008): Die Konzeptualisierung von Politik als Krieg - Metaphern in der politischen Berichterstattung. In: SASI / Studentische Arbeitspapiere zu Sprache und Interaktion 12. http://noam.uni-muenster.de/SASI (9.1.2018).

Küster, Rainer (1978): Militärmetaphorik im Zeitungskommentar: Darstellung und Dokumentation an Leitartikeln der Tageszeitungen Die Welt und Süddeutsche Zeitung. Göppingen: Kümmerle. 
Küster, Rainer (1983): Politische Metaphorik. In: Sprache und Literatur in Wissenschaft und Unterricht 51, S. 30-45.

Lakoff, George (2006): Ženy, oheň a nebezpečné věci. Co kategorie vypovídají o naší mysli. [Women, Fire and Dangerous Things. What Categories Reveals about the Mind.] Praha: Triáda.

Lakoff, George - Johnson, Mark (2002): Metafory, kterými žijeme. [Metaphers We Live by.] Brno: Host.

La Roche, Walther von (1992): Einführung in den praktischen Journalismus (13., neu bearb. Aufl.). München: List.

Lüger, Heinz-Helmut (1995): Pressesprache (2., neu bearb. Aufl.). Tübingen: Niemeyer.

Malá, Jiřina (2003): Stilistische Funktionen der Phraseologismen in publizistischen Textsorten. In: Korčáková, Jana - Beyer, Jürgen (Hrsg.): Königgrätzer Linguistik- und Literaturtage. Hradec Králové: Gaudeamus, S. 307-312.

Malá, Jiřina (2008): Metaphern und Idiome als textstilistische Mittel in der Textsorte Filmrezension. Dargestellt an Filmrezensionen aus den Wochenmagazinen Der Spiegel und FOCUS. In: Brünner Beiträge zur Germanistik und Nordistik. Sborník prací filozofické fakulty brněnské univerzity, R 13, S. 45-55.

Malá, Jiřina (2009): Stilistische Textanalyse: Grundlagen und Methoden. Brno: Masarykova univerzita.

Mast, Claudia (Hrsg.) (1994): ABC des Journalismus. Ein Leitfaden für die Redaktionsarbeit (7., völlig neue Ausgabe). Konstanz: Ölschläger.

Molly, Helmut (2002): Auf dem Weg zur deutschen Einheit. Zur sprachlichen Evidenz einer politischen Metapher. In: Zeitschrift für germanistische Linguistik 30, 3, S. 356-395.

Mutz, Katrin (2010): Helge Skirl / Monika Schwarz-Friesel (2007): Metapher, Heidelberg, Universitätsverlag Winter (Kurze Einführungen in die germanistische Linguistik, Band 4), $100 \mathrm{~S}$. In: metaphorik.de 18, S. 107-113. http://www.metaphorik.de/sites/www.metaphorik.de/files/ journal-pdf/18_2010_rezensionskirl-schwarzfriesel.pdf (1.7.2019).

Nešpor, Václav (1998): Dějiny města Olomouce. [Geschichte der Stadt Olmütz.] Reprint der Originalausgabe von 1936. Olomouc: Votobia.

Niehr, Thomas (2014): Einführung in die Politolinguistik. Gegenstand und Methoden. Göttingen: Vandenhoeck \& Ruprecht.

Nowag, Werner - Schalkowski, Edmund (1998): Kommentar und Glosse. Konstanz: UVK Medien. Palm, Christine (1995): Phraseologie. Eine Einführung. Tübingen: Narr.

Piirainen, Elisabeth (2007): Phrasemes from a cultural semiotic perspective. In: Burger, Harald [u.a.] (Hrsg.): Phraseologie / Phraseology. Ein internationales Handbuch zeitgenössischer Forschung. Bd. 1. Berlin: de Gruyter, S. 208-219.

Piirainen, Elisabeth (2011): Weit verbreitete Idiome in kulturhistorischen Bezügen: zum Verbreitungsmodus einiger Idiomgruppen. In: Das Wort. Germanistisches Jahrbuch Russland 2010. Bonn: DAAD, S. 167-181.

Polenz, Peter von (1991): Deutsche Sprachgeschichte vom Spätmittelalter bis zur Gegenwart. Bd. I. Einführung, Grundbegriffe, Deutsch in der frühbürgerlichen Zeit. Berlin: de Gruyter.

Reger, Harald (1980): Die Metaphorik in der konventionellen Tagespresse. In: Reger, Harald: Metaphern und Idiome in szenischen Texten, in der Werbe- und Pressesprache. Hamburg: Helmut Buske Verlag, S. 76-96. (Urspr. in: Muttersprache 1977, 4, S. 259-279.)

Reumann, Kurt (2004): Journalistische Darstellungsformen. In: Noelle-Neumann, Elisabeth Schulz, Winfried - Wilke, Jürgen (Hrsg.): Das Fischer Lexikon Publizistik / Massenkommunikation (3. Aufl.). Frankfurt a. M.: Fischer, S. 126-152.

Röhrich, Lutz (2004): Lexikon der sprichwörtlichen Redensarten (7. Aufl.). Freiburg: Herder.

Rykalová, Gabriela (2008): Entwicklungstendenzen in der Tagespresse. Dargestellt an journalistischen Textsorten der deutschsprachigen Zeitungen. Saarbrücken: SVH. 
Schemann, Hans (2011): Deutsche Idiomatik. Wörterbuch der deutschen Redewendungen im Kontext (2. Aufl. mit vollst. überarb. Einführung). Berlin: de Gruyter. https://books.google. cz/books?id=oduMIx5bOF0C\&hl=cs\&source=gbs_navlinks_s (5. 7. 2019).

Schmitt, Rudolf (2004). Diskussion ist Krieg, Liebe ist eine Reise, und die qualitative Forschung braucht eine Brille. Review Essay. In: Forum Qualitative Sozialforschung 5, 2, Art. 19. http:// nbn-resolving.de/urn:nbn:de:0114-fqs0402190 (28. 6. 2019).

Stöber, Rudolf (2005): Deutsche Pressegeschichte. Von den Anfängen bis zur Gegenwart (2., überarb. Aufl.). Konstanz: UVK. https://books.google.cz/books?id=4Yppo08pQBUC\&hl= cs\&source=gbs_navlinks_s (28.6.2019).

Thurmair, Maria (2002): Der Harald Juhnke der Sprachwissenschaft. Metaphorische Eigennamenverwendungen. In: Deutsche Sprache 30, 1, S. 1-27.

URL1 = Wallenstein. In: Wikipedia, Die freie Enzyklopädie (Bearbeitungsstand 18. 6. 2019). https://de.wikipedia.org/wiki/Wallenstein (8. 7. 2019).

Weydt, Harald (1992): Metaphernfunktionen in Texten. In: Ágel, Vilmos - Hessky, Regina (Hrsg.): Offene Fragen - offene Antworten in der Sprachgermanistik. Tübingen: Niemeyer, S. 35-44.

Mgr. Michaela Kaňovská, Ph.D. / michaela.kanovska@upol.cz

Univerzita Palackého, Filozofická fakulta, Katedra germanistiky,

Křížkovského 10, 77180 Olomouc, CZ

This work can be used in accordance with the Creative Commons BY-SA 4.0 International license terms and conditions (https://creativecommons.org/licenses/by-sa/4.0/legalcode). This does not apply to works or elements (such as image or photographs) that are used in the work under a contractual license or exception or limitation to relevant rights 
